\title{
Charles Bonnet Syndrome as a Rare Complication in an Ischaemic Stroke
}

\author{
Helder Santos, ${ }^{1}$ Pedro Agrela, ${ }^{2}$ Catarina Parente, ${ }^{3}$ Kelly Lopes, ${ }^{4}$ Sara Santos, ${ }^{3}$ António Cardoso ${ }^{3}$ and Fátima Campante ${ }^{3}$ \\ 1. Department of Cardiology, Centro Hospitalar Barreiro Montijo EPE, Barreiro, Portugal; 2. General Practice, Centro de Saúde Bom Jesus, \\ Funchal, Portugal; 3. Department of Internal Medicine, Centro Hospitalar Barreiro Montijo EPE, Barreiro, Portugal; 4. Department of \\ Pneumology, Centro Hospitalar Barreiro Montijo EPE, Barreiro, Portugal.
}

DOl: https://doi.org/10.17925/ENR.2018.13.2.120

\begin{abstract}
$\mathrm{C}$ harles Bonnet Syndrome (CBS) is a rare cause of complex visual hallucinations (VH) in individuals with preserved cognitive status. We report a case of an elderly patient with $\mathrm{VH}$, diplopia and headaches for 1 week, with preserved visual acuity and without any strength or focal neurological findings. Computed tomography revealed a subacute ischaemic stroke with temporal and occipital involvement. The patient was admitted to the Department of Internal Medicine where neurological, ophthalmological and psychiatric primary disease were ruled out, as well as dementia. CBS was assumed as a result of a subacute ischaemic stroke. Valproic acid was initiated to treat the symptoms, which resolved the $\mathrm{VH}$. After 3 months of follow up, the patient remained completely recovered without any dementia signs. CBS is a benign disease, usually controlled without pharmacological therapy and not associated with a psychiatric disease; nevertheless, its association with dementia is not clear.
\end{abstract}

\section{Keywords}

Stroke, visual hallucinations, Charles Bonnet syndrome, dementia, elderly, valproic acid

Disclosures: Helder Santos, Pedro Agrela, Catarina Parente, Kelly Lopes, Sara Santos, António Cardoso and Fátima Campante have no conflicts of interest to declare in relation to this article.

Review Process: Double-blind peer review. Authorship: All named authors meet the criteria of the International Committee of Medical Journal Editors for authorship for this manuscript, take responsibility for the integrity of the work as a whole and have given final approval for the version to be published.

Compliance with Ethics: All procedures were followed in accordance with the responsible committee on human experimentation and with the Helsinki Declaration of 1975 and subsequent revisions, and informed consent was received from the patient involved in this case study.

open Access: This article is published under the Creative Commons Attribution Non-commercial License, which permits any non-commercial

use, distribution, adaptation and reproduction provided the original author(s) and source are given appropriate credit. (C) The Authors 2018.

Received: 25 October 2018

Accepted: 28 November 2018

Citation: European Neurological Review. 2018;13(2):120-2

Correspondence: Helder Santos, Cardiology Department, Centro Hospitalar Barreiro-Montijo, Avenida Movimento das Forças Armadas,

Barreiro, Portugal. E: helder33689@gmail.com

Support: No funding was received in

the publication of this article.
Charles Bonnet syndrome (CBS) is characterised by the presence of visual hallucinations (VH) and visual sensory deprivation in individuals with preserved cognitive status and without a history of psychiatric illness. ${ }^{1} \mathrm{CBS}$ is a rare, underdiagnosed and under-recognised syndrome, which was first described in 1769 by Charles Bonnet, who observed this phenomenon in his grandfather.'

The prevalence of CBS is not consistent between authors. Some studies have stated that $0.47 \%$ of the general population suffer from CBS - this number can increase to around 15-30\% when referring to patients with visual impairment. The disparity in CBS prevalence can be explained by the absence of clear diagnostic criteria, the necessity of different specialties to recognise or exclude other disorders and the incapability of patients to express the symptoms. ${ }^{2,3}$ CBS usually occurs in elderly people with compromised visual function and can be a rare manifestation of an injured visual pathway. Secondary lesions to multiple sclerosis, ischaemic stroke, temporal arteritis and meningiomas can explain the syndrome. This case report presents a variant of CBS as consequence of an ischaemic stroke in left occipitotemporal regions. In our view, the particularities and variations of CBS should be widespread, as it can be the only manifestation of an ischaemic stroke that requires appropriate and immediate management.

\section{Case report}

We describe a 74-year-old woman, Caucasian and independent in terms of daily activities. Her past medical history included:

- arterial hypertension, which was well controlled with valsartan and hydrochlorothiazide;

- thyroid nodules with a grade II Bethesda classification after an internal medicine consultation, plus controlled thyroid function; and

- grade II gonarthrosis with bilateral Baker's cysts.

She presented at our emergency department (ED) with a sudden episode of $\mathrm{VH}$ (only on the right visual field), diplopia, nausea and headaches (described as 'the worst headaches of her life'), which started the previous week. The hallucinations were vivid, clear and intermittent, lasting from 30 minutes to an hour, with small intervals from 5-15 minutes, occurring more frequently while watching television or other activities that required a greater visual focus. The patient described the visions as 'things that should not be there' - like 'cars, animals and people' - being able to describe the colours, shapes and motions; nevertheless, she could not recognise people's faces. She denied any decrease of muscular strength and sensibility. There was no report of personal mental disease, substance abuse, trauma or fever. The patient visited an ophthalmologist the previous day, due to the visual impairment, who could not find any ocular variations responsible for the neurological segment manifestations. 
Upon physical examination, the patient presented conscious, alert and oriented to person, time and event. Dysarthria and strength or focal findings were not present upon neurological examination. Cardiopulmonary auscultation had no abnormal shifts and her vital signs only displayed isolated systolic arterial hypertension (systolic $170 \mathrm{mmHg}$; diastolic $90 \mathrm{mmHg}$ ), with a rhythmic pulse rate of 85 beats/minute, temperature of $36.2^{\circ} \mathrm{C}$ and an oxygen saturation of $99 \%$ while breathing ambient air. Neurological and optical examination showed right-side homonymous hemianopsy, in which the complex VH were limited. She presented no cognitive deficits and a Mini-Mental State Examination of $30 / 30$ with no focal findings on neurological examination. Visual acuity was considered normal and there were no auditory hallucinations or any other pathological manifestations on the physical exam.

Blood work did not show any significant findings - only revealing a discrete alteration in serum glucose (155 mg/dL), sodium (135 mmol/L) and C-reactive protein $(14.4 \mathrm{mg} / \mathrm{L})$. A cranial-encephalic computed tomography (CE-CT) revealed a hypodense cortical lesion in the left temporal and occipital region, suggesting a subacute ischaemic stroke.

The patient was then admitted in to the Internal Medicine Department for an aetiologic study of ischaemic stroke with recurrent VH. A complete neurological study excluded neurological disorders and any psychiatric illness. Electroencephalogram did not detect epileptic activity responsible for the symptoms. After a complete study, diagnosis of CBS secondary to occipitotemporal ischaemic stroke was assumed. The hallucinations continued throughout hospitalisation duration and were only gradually reverted after medical therapy introduction. Therapy with valproic acid was initiated and after 2 days, the patient's hallucinations disappeared. Follow-up after 3 months showed the resolution of hallucinations and the complete recovery of the patient.

\section{Discussion}

CBS is defined by $\mathrm{VH}$, visual sensory deprivation and a preserved cognitive status. This triad occurs mostly due to senile macular degeneration and other anterior visual pathway dysfunction in patients of advanced age. In fewer patients, it can occur as result of an ischaemic stroke that affects optical neural pathways, the occipital visual cortex or both. In our case, the last was observed with an ischaemic stroke affecting the area between the temporal and occipital cortex. Although being rare, observations of migraine with complex VH have been registered, occurring days to weeks after the stroke and disappearing in weeks. ${ }^{2-4}$

VH are vivid, complex and represent daily objects and people; some patients described dead people. Usually the hallucinations tend to repeat and patients recognise the images as unreal and uncontrolled. However, in some cases, these images can become realistic, thus inducing the patient to believe that the scene is real and causing reactions according to the scene. ${ }^{5,6}$ The frightening prospect of considering $\mathrm{VH}$ as a mental disorder can discourage the patient from expressing them as a symptom, thus hindering the correct diagnosis.

Complex hallucinations are defined as illusory images of animals, vehicles, objects, people and faces, sounds or smells in the absence of an external stimulus. On the other hand, simple hallucinations are represented as coloured shapes or as a pattern. Both can be manifested in CBS, but only the complex hallucinations define the disorder, with the visual component being more frequently displayed and considered neutral, despite other cases reporting visual and auditory hallucinations. The hallucinations are present in conscious, alert and awake patients, without signs of dementia or altered mental function. ${ }^{2,7.8}$ Despite generally having a time inferior to 12 months, the duration of the hallucinations can diverge from seconds to being permanent with years of evolution and can be categorised as episodic, cyclic or chronic. ${ }^{9}$ Any trigger factor can promote hallucinations and in some individuals it is possible to identify a specific trigger. ${ }^{3}$ In our case, the patient mentioned having episodes more frequently while watching television or any other activity that required more visual focus. Some authors proposed risk factors such as: age (more than 64 years old), social isolation, low cognitive function, past history of stroke and deprived bilateral visual acuity.5.10

This case presents some characteristic traces of CBS, such as VH and the absence of psychiatric and neurologic illness, such as dementia. Visual acuity, other than a homonymous hemianopsia, was preserved, with an ischaemic stroke being aetiologically responsible for the symptoms. Some of the risk factors, like the social isolation, do not apply in this case, since the patient lived with her daughter and was independent in her daily activities.

Diagnosis is generally made in elderly people, yet at this age hallucinations can have a neurological aetiology, representing early manifestations of Alzheimer's disease, dementia, Parkinson's disease, delirium, sensory or sleep deprivation and psychosis. ${ }^{6}$ These require a neurological study to detect any early manifestation, with some authors suggesting that CBS should be considered an early marker of dementia, since patients frequently develop the disease during long-term follow up. ${ }^{11}$ The subject of this study was elderly, yet at this point no signs of dementia were observed. Regardless, we cannot exclude the emergence of dementia in a medium- or long-term follow up.

This syndrome was also reported in cases of peduncular hallucinosis and multiple sclerosis. Epilepsy can be manifested as VH; however, these are usually simple, transitory and accompanied by seizures. It is important to remember that complex VH imply the involvement of the temporal cortex. In contrast, parietal cortex lesions may be one of the causes of complex $\mathrm{VH}$, but concomitant occipital cortex involvement must be present. ${ }^{2-4}$ In our report case, the electroencephalogram did not identify any seizure activity nor other clinical examinations suggested other diseases responsible for the clinical manifestations.

The pathophysiology of CBS is unclear, but possible explanations were postulated. One of the most accepted theories proposes that lesions in the visual pathway produce and transmit abnormal signals to the visual cortex. These signals, when fused with a normal visual stimulus, cause the so-called VH. An alternative theory argues that a reduction in visual impairment leads to the formation of spontaneous images, resulting in the $\mathrm{VH}$. Other factors that can influence the $\mathrm{VH}$ manifestations are social isolation, reduced cerebral perfusion, hearing loss and other psychological factors, according to the last theory. $3,5,7,12$

In the present case, cerebral dysfunction was not the aim of the study since there are no scientific reports highlighting its importance to CBS diagnosis. Patients with CBS normally undergo a CT scan and, in some cases, magnetic resonance imaging (MRI); however, these techniques do not identify any abnormalities. Patients with VH in the MRI can show hyperactivity on the occipital cortex, though, to the authors' knowledge, there are no scientific reports regarding the significance of these findings. On the other hand, a single photon emission CT performed in patients with $\mathrm{VH}$ may reveal a transitional hyperperfusion on the temporal cortex. Despite these findings suggesting an occipital cortex dysfunction, results were reported in fewer than 10 patients. ${ }^{13}$ 
CBS is a diagnosis of exclusion, so the management of these patients implies exclusion of other pathologies known to cause VH. Only after a full characterisation of other possibilities can the physician assume CBS as a diagnosis, explaining to the patient and family that hallucinations are a benign process and not caused by a psychiatric disorder. When visual impairment and hallucinations are present, patients should be evaluated by an ophthalmologist, in order to determine if there are any ocular findings or surgery indications, and by a psychiatrist to understand if the hallucinations are formed with a psychiatric basis. When any other disorder can explain the symptoms, primary disease therapy is recommended. Since there are no guidelines to regulate CBS treatment nor any therapy indicated, the known pharmacological therapy is based on case reports and a small case series in the literature. Due to this, we can find reports of CBS therapy with antipsychotics, anticonvulsants and selective serotonin reuptake inhibitors. This pathology generally represents a benign event; therefore, the pharmacological therapy should be postponed and reserved for cases associated with distress or a symptomatic phase that does not allow the individual to maintain his or her daily activity. $2,5,12$ Our patient was experiencing VH for 2 weeks, prior to first contact in the ED, and continued for the 2 weeks after, while performing a study for the ischaemic stroke. At that time, therapy with valproic acid was initiated, the hallucinations gradually decreased, and in end of a 3-month follow up, the patient presented without any VH.

CBS is a rare etiology of $\mathrm{VH}$ and may not be identified by many healthcare professionals (HCPS) since the search for $\mathrm{VH}$ is not frequent in stroke patients. $\mathrm{VH}$ in elderly individuals can occur due to neurological, ophthalmological and psychiatric disorders and other acute complications such as ischaemic stroke, which requires a careful and fast response from HCPS. The under-diagnosis of CBS can be related to the patient's fear of being diagnosed with a mental disorder, hiding the symptoms from themselves. Therefore, it's crucial that physicians recognise this syndrome in early phases and provide both patient and family with proper information about the disease. Since some authors consider this syndrome as an early sign of dementia, a follow-up is recommended. The hallucinations can disappear over time, yet, in some cases, pharmacological therapy is required. In our case, the early recognition and treatment of patient's condition had a positive impact on her quality of life. We believe that prompt clinical recognition of CBS can prevent healthcare overuse and also neurological and/or cardiovascular complications related to stroke. $]$
1. Chaudhuri A. Charles Bonnet syndrome: an example of cortical dissociation syndrome affecting vision? I Neurol Neurosurg Psychiatry. 2000;69:704

2. Pang L. Hallucinations experienced by visually impaired Charles Bonnet syndrome Optom Vis Sci. 2016:93:1466

3. Menon GJ, Rahman I, Menon SJ, Dutton GN. Complex visual hallucinations in the visually impaired: the Charles Bonnet Syndrome. Surv Ophthalmol. 2003;4:58-72.

4. Senadim S, Balcik Z, Uygun E, et al. Visual hallucinations and ischemic stroke: review of 5 cases. I Neurol Neurophysiol. $2017 ; 8: 2$

5. Nair AG, Nair AG, Shah BR, Gandhi RA. Seeing the unseen: Charles Bonnet syndrome revisited. Psychogeriatrics. 2015;15:204-8.
6. Vukicevic M, Fitzmaurice K. Butterflies and black lacy patterns: the prevalence and characteristics of Charles Bonnet hallucinations in an Australian population. Clin Exp Ophthalmol. 2008:36:659-65.

7. Schadlu AP, Schadlu R, Shepherd JB. Charles Bonnet syndrome: a review. Curr Opin Ophthalmol. 2009;20:219-22.

8. Kester EM. Charles Bonnet syndrome: case presentation and Kester EM. Charles Bonnet syndrome: case p
literature review. Optometry. 2009;80:360-6.

9. Santos-Bueso E, Sáenz-Francés F, Serrador-García M, et al. Prevalence and clinical characteristics of Charles Bonnet syndrome in Madrid, Spain. Eur J Ophthalmol 2014;24:960-3

10. Teunisse RJ, Cruysberg JR, Verbeek A, Zitman FG. The Charles Bonnet syndrome: a large prospective study in the Netherlands: a study of the prevalence of the Charles Bonnet syndrome and associated factors in 500 patients attending the University Department of Ophthalmology at Nijmegen Br J PSychiatry 1995;166:254-7.

11. Lapid MI, Burton MC, Chang MT, et al. Clinical phenomenology and mortality in Charles Bonnet syndrome. J Geriatr Psychiatry Neurol. 2013;26:3-9.

12. O'Farrell L, Lewis S, McKenzie A, Jones L. Charles Bonnet Syndrome: a review of the literature. Journal of Visual Impairment \& Blindness. 2010;104:267-74.

13. Kazui H, Ishii R, Yoshida T, et al. Neuroimaging studies in patients with Charles Bonnet Syndrome. Psychogeriatrics. 2009;9:77-84 